\title{
An active Mitochondrial Complex II Present in Mature Seeds Contains an Embryo-Specific Iron-Sulfur Subunit Regulated by ABA and bZIP53 and Is Involved in Germination and Seedling Establishment
}

\author{
Franko Restovic ${ }^{1}$, Roberto Espinoza-Corral' ${ }^{1}$, Isabel Gómez ${ }^{1}$, Jesús Vicente-Carbajosa ${ }^{2}$ \\ and Xavier Jordana ${ }^{1 *}$ \\ ' Departamento de Genética Molecular y Microbiología, Facultad de Ciencias Biológicas, Pontificia Universidad Católica de \\ Chile, Santiago, Chile, ${ }^{2}$ Centro de Biotecnología y Genómica de Plantas - UPM-INIA, Campus de Montegancedo, \\ Universidad Politécnica de Madrid, Madrid, Spain
}

OPEN ACCESS

Edited by:

Nicolas Rouhier,

University of Lorraine, France

Reviewed by:

Uener Kolukisaoglu,

University of Tübingen, Germany

Pascal Rey,

Commissariat à l'Energie Atomique et aux Energies Aternatives, France

*Correspondence:

Xavier Jordana

xjordana@bio.puc.cl

Specialty section:

This article was submitted to

Plant Physiology,

a section of the journal

Frontiers in Plant Science

Received: 01 September 2016 Accepted: 14 February 2017

Published: 28 February 2017

Citation:

Restovic F, Espinoza-Corral R, Gómez I, Vicente-Carbajosa J and

Jordana X (2017) An active

Mitochondrial Complex II Present

in Mature Seeds Contains an

Embryo-Specific Iron-Sulfur Subunit Regulated by ABA and bZIP53 and is Involved in Germination and Seedling Establishment.

Front. Plant Sci. 8:277. doi: 10.3389/fpls.2017.00277
Complex II (succinate dehydrogenase) is an essential mitochondrial enzyme involved in both the tricarboxylic acid cycle and the respiratory chain. In Arabidopsis thaliana, its iron-sulfur subunit (SDH2) is encoded by three genes, one of them (SDH2.3) being specifically expressed during seed maturation in the embryo. Here we show that seed SDH2.3 expression is regulated by abscisic acid (ABA) and we define the promoter region $(-114$ to +49$)$ possessing all the cis-elements necessary and sufficient for high expression in seeds. This region includes between -114 and -32 three ABRE (ABAresponsive) elements and one RY-enhancer like element, and we demonstrate that these elements, although necessary, are not sufficient for seed expression, our results supporting a role for the region encoding the $5^{\prime}$ untranslated region $(+1$ to +49$)$. The $S D H 2.3$ promoter is activated in leaf protoplasts by heterodimers between the basic leucine zipper transcription factors bZIP53 (group S1) and bZIP10 (group C) acting through the ABRE elements, and by the B3 domain transcription factor ABA insensitive 3 (ABI3). The in vivo role of bZIP53 is further supported by decreased SDH2.3 expression in a knockdown bzip53 mutant. By using the protein synthesis inhibitor cycloheximide and sdh2 mutants we have been able to conclusively show that complex II is already present in mature embryos before imbibition, and contains mainly SDH2.3 as iron-sulfur subunit. This complex plays a role during seed germination sensu-stricto since we have previously shown that seeds lacking SDH2.3 show retarded germination and now we demonstrate that low concentrations of thenoyltrifluoroacetone, a complex II inhibitor, also delay germination. Furthermore, complex II inhibitors completely block hypocotyl elongation in the dark and seedling establishment in the light, highlighting an essential role of complex II in the acquisition of photosynthetic competence and the transition from heterotrophy to autotrophy.

Keywords: mitochondrial respiratory complex, succinate dehydrogenase, iron-sulfur subunit, abscisic acid, ABI3, bZIPs, Arabidopsis seed germination, seedling establishment 


\section{INTRODUCTION}

Mitochondrial Complex II or SDH (succinate:ubiquinone oxidoreductase, EC 1.3.5.1) plays a central role in mitochondria as the only enzyme of two fundamental metabolic pathways: the TCA cycle and the respiratory chain. This complex associated to the inner mitochondrial membrane catalyzes the transfer of electrons from succinate to ubiquinone, generating fumarate and ubiquinol. Complex II is the simplest of the ETC complexes, and in most organisms, it contains four subunits (Yankovskaya et al., 2003; Sun et al., 2005). The flavoprotein (SDH1) contains the succinate binding and oxidation site, and interacts with the iron-sulfur protein $(\mathrm{SDH} 2)$, which contains three nonheme iron-sulfur centers mediating the transfer of electrons to the membrane. The peripheral (matrix side) SDH1-SDH2 subcomplex is anchored to the membrane by two small integral membrane proteins (SDH3 and $\mathrm{SDH} 4$ ), which contain the ubiquinone binding and reduction site (Yankovskaya et al., 2003; Sun et al., 2005). Interestingly, additional subunits of unknown function have been described for plant Complex II (Millar et al., 2004; Huang and Millar, 2013).

Complex II subunits are all nuclear-encoded in Arabidopsis thaliana (Figueroa et al., 2001, 2002; Millar et al., 2004). Surprisingly, several of the complex II subunits are encoded by more than one gene in Arabidopsis. For instance, we have reported that two genes, named SDH1.1 and SDH1.2, encode the flavoprotein and that SDH1.1 is the main flavoprotein subunit found in Complex II and is essential for gametophyte development (León et al., 2007). Three genes, designated SDH2.1 (At3g27380), SDH2.2 (At5g40650), and SDH2.3 (At5g65165), encode the iron-sulfur subunit. Considering that in most organisms there is a single $\mathrm{SDH} 2$ gene, the presence of three genes in Arabidopsis raises interesting questions about their roles during plant development. The three $\mathrm{SDH} 2$ proteins would be functional, since they are highly conserved when compared with their homologues in other organisms and contain the cysteine motifs involved in binding the three iron-sulfur clusters essential for electron transport (Figueroa et al., 2001). SDH2.1 and SDH2.2 genes likely arose via a relatively recent duplication event and are redundant. Indeed, both genes have similar exon-intron structures, encode nearly identical proteins and are similarly expressed in all organs from adult plants (Figueroa et al., 2001; Elorza et al., 2004). Moreover, the knockouts of SDH2.1 and $S D H 2.2$ do not have any phenotype, and we have been unable to obtain double sdh2.1/sdh2.2 homozygous mutants (Elorza et al., 2004 and unpublished results). In contrast, SDH2.3 exonintron structure is completely different from that of $S D H 2.1$ and $S D H 2.2$, the encoded protein is only $67 \%$ similar to SDH2.1 and SDH2.2, and $S D H 2.3$ is specifically expressed in the embryo during seed maturation. Indeed, Elorza et al. (2006) showed that $S D H 2.3$ mRNA begins to accumulate in maturing embryos, is

Abbreviations: $\mathrm{ABA}$, abscisic acid; $\mathrm{ABI}$, $\mathrm{ABA}$ insensitive 3; $\mathrm{ABRE}, \mathrm{ABA}$ responsive element; AuxRE, auxin responsive element; bZIP, basic leucine zipper transcription factor; DOF, DNA binding with one zinc finger; ETC, electron transport chain; GUS, $\beta$-glucuronidase; NAN, neuraminidase; SDH, succinate dehydrogenase; SSP, seed storage proteins; TCA, tricarboxylic acid; TTFA, thenoyltrifluoroacetone; UTR, untranslated region. abundant in dry seeds and declines during germination and early post-germinative growth.

SDH2.3 highly specific expression during embryo maturation raises interesting questions about the regulatory mechanism. Using promoter fusions to the GUS reporter gene, we first showed that $S D H 2.3$ expression is transcriptionally regulated (Elorza et al., 2006). Then, using mutated promoters, we demonstrated that three ABRE (abscisic acid responsive) elements and a RY-like enhancer element are necessary for its embryo-specific transcriptional regulation (Roschzttardtz et al., 2009). ABRE and RY elements have been implicated in the seed-specific expression of SSP genes and late embryogenesis abundant proteins (LEAs) genes (Parcy et al., 1994; Busk and Pagès, 1998; Nambara and Marion-Poll, 2003). Furthermore, three master regulators of seed maturation belonging to the B3 domain transcription factors family, ABSCISIC ACID INSENSITIVE 3 (ABI3), FUSCA3 (FUS3), and LEAFY COTYLEDON 2 (LEC2) (Santos-Mendoza et al., 2008), control SDH2.3 expression in planta (Roschzttardtz et al., 2009). In contrast, although ABRE elements are known targets for transcription factors of the basic leucine zipper (bZIP) family, the in vivo role of bZIP transcription factors in $S D H 2.3$ regulation was not assessed. Here we show that bZIP53 controls SDH2.3 expression in planta and that bZIP53/bZIP10 heterodimers are able to activate the $S D H 2.3$ promoter. Furthermore, we demonstrated that ABA controls seed SDH2.3 expression.

$S D H 2.1$ and SDH2.2 are expressed at very low levels during seed maturation and in mature seeds and their expression is induced during germination and early post-germinative growth (Elorza et al., 2006; Roschzttardtz et al., 2009). Thus, data suggest that a SDH2.3 containing Complex II may have a role at these early developmental steps, and that SDH2.3 is gradually exchanged for SDH2.1/2.2 as the iron-sulfur subunit. Consistently, here we show using single sdh2.1 and sdh2.3 mutants, and double $s d h 2.1 / s d h 2.3$ mutants, that a Complex II containing mainly the iron-sulfur subunit $\mathrm{SDH} 2.3$ is already present in mature dry seeds, before imbibition, and that this Complex II with SDH2.3 has an important but not essential role at early stages of plant development. In contrast, Complex II is essential for chloroplast development and seedling establishment.

\section{MATERIALS AND METHODS}

\section{Plant Material and Growth Conditions}

All $A$. thaliana plant materials used were in the Columbia (Col0) background. Seeds of the T-DNA insertion mutants bzip10 (SALK_106031, NASC ID: N606031), bzip25 (SALK_119931, NASC ID: N619931) and bzip53 (SALK_069883, NASC ID: N569883) were obtained from the Nottingham Arabidopsis Stock Centre, and homozygous mutants have been previously identified (Weltmeier et al., 2006). bzip53 is a knockdown line with the T-DNA inserted upstream of the ATG initiation codon, bzip10 and bzip25 are knockout lines. Mutants in ABA biosynthesis were also obtained from the Nottingham Arabidopsis Stock Centre: $a b a 2-1$ and aba2-3 have impaired xanthoxin oxidation, aba3-1 
and aba3-2 have impaired abscisic aldehyde oxidation (Schwartz et al., 1997; Barrero et al., 2006).

Two homozygous sdh2.3 insertional mutants, called dSpm (sdh2.3-1) and DsLox (sdh2.3-2), and two homozygous sdh2.1 insertional mutants, called GARLIC (sdh2.1-1) and SALK (sdh2.1-2), have been previously characterized (Elorza et al., 2004; Roschzttardtz et al., 2009). The sdh2.3-1 mutant was crossed with either the $s d h 2.1-1$ mutant or the $s d h 2.1-2$ mutant. F1 seeds from the $s d h 2.3-1 / s d h 2.1-2$ cross were germinated on Basta ${ }^{\circledR}$ and kanamycin, and F1 seeds from the sdh2.3-1/sdh2.1-1 cross were germinated on Basta ${ }^{\circledR}$. To isolate homozygous double mutants in next generations, genotyping was performed as described (Elorza et al., 2004; Roschzttardtz et al., 2009).

Seeds were surface-sterilized, stratified for $48 \mathrm{~h}$ at $4^{\circ} \mathrm{C}$ in the dark and then sown on one-half-concentrated MS medium with $0.8 \%(\mathrm{w} / \mathrm{v})$ agar. After 2 weeks at $22^{\circ} \mathrm{C}$ under long-day conditions (16-h/8-h day/night cycle), seedlings were transferred to soil and grown either under long-day conditions or short-day conditions (8-h-light/16 h-dark cycle).

To measure hypocotyl growth in the dark and assay germination, seeds were surface-sterilized, sown on halfconcentrated MS agar, and stratified for $48 \mathrm{~h}$ at $4^{\circ} \mathrm{C}$. TTFA, carboxin, malonate, or sodium azide were added as indicated in the Figures. For hypocotyl measurements plates were placed vertically in the dark for 6 days, at $22^{\circ} \mathrm{C}$. After scanning in an Epson Perfection v700 Photoscanner, images were analyzed with Image $J$ software ${ }^{1}$. For germination assays, plates were incubated horizontally at $22^{\circ} \mathrm{C}$ under long-day conditions, and germination was scored at different times based on radicle protrusion. Seedling establishment was evaluated at 10 days based on the emergence of true leaves.

\section{Constructs for Transformation of Arabidopsis Plants}

A construct containing the $S D H 2.3$ promoter and 5' UTR fused to the GUS coding sequence $(-223 /+49$, numbers in relation to the $S D H 2.3$ transcription initiation site) has been previously described (P3 construct in Elorza et al., 2004). To shorten the promoter, forward primer sdh $2.3 \mathrm{~F}$ and reverse primer sdh $2.3 \mathrm{R}$ (see Supplementary Table S1 for oligonucleotides) were used to amplify by PCR the region between -114 and +49 , using Arabidopsis genomic DNA as template. Putative AuxRE and DOF elements were mutagenized by $\mathrm{PCR}$, using the $\mathrm{P} 3$ construct as template. Two PCRs were carried for each mutant with the same template. For the promoter mutated in the AuxRE element (mAuxRE), one amplification was done with primers sdh2.3F and mAuxRE- $\mathrm{R}$ and the other was performed with primers sdh2.3R and mAuxRE-F. A mixture of both amplification products was used as a template for a third PCR with primers sdh $2.3 \mathrm{~F}$ and $s \mathrm{dh} 2.3 \mathrm{R}$. The same procedure was employed to obtain constructs mutated in DOF (mDOF) or in both AuxRE and DOF elements (mAuxRE/mDOF), using sdh2.3F, sdh2.3R, and the following "mutated" primers: for mDOF, forward primer mDOF-F and reverse primer mDOF-R, and for the double mutant forward primer mAUXRE/mDOF-F and reverse

\footnotetext{
${ }^{1}$ http://rsb.info.nih.gov/ij/
}

primer mAUXRE/mDOF-R. PCR products $(-114 /+49$ wild type and mutated constructs) were cloned into pGEM-T plasmid (Promega), and the DNA fragments obtained by digestion with Bam HI and NcoI were ligated into pCAMBIA $1381^{2}$.

Three PCRs were performed to fuse the -114 to -32 region of the $S D H 2.3$ promoter to the minimal cauliflower mosaic virus (CaMV) $35 \mathrm{~S}$ promoter. First, we amplified the $-114 /-32$ fragment using the P3 construct as template, forward primer sdh2.3F' and reverse primer RYR. Then, we amplified the CaMV35S minimal promoter $(-64$ to +41$)$ using forward primer sdh2.3/35S, reverse primer GUS35S-R, and pBI121 (Clontech) as template. Finally, we used as template a mixture of both amplification products for a third PCR with primers sdh $2.3 \mathrm{~F}$ ' and GUS35S-R. The same procedure was followed to fuse the -223 to -32 region of the $S D H 2.3$ promoter to the CaMV35S minimal promoter, using forward primer sdh2.3F2 in first and third PCRs. PCR products were cloned into PGEM-T, recombinant plasmids were digested with $E c o R I$ and $\mathrm{NcoI}$ and inserts were ligated into pCAMBIA1381.

To fuse the fragment upstream of the $S D H 2.3$ transcription initiation site $(-114$ to -1$)$ to the CaMV35S 5'UTR, four serial PCRs were carried out. In the four PCRs the same forward primer $\left(\mathrm{sdh} 2.3 \mathrm{~F}^{\prime}\right)$ was used, in combination with different partially overlapping reverse primers designed to reconstitute the 5'UTR: 35S-5UTR1-R, 35S-5UTR2-R, 35S-5UTR3-R, and finally primer GUS35S-R. Final PCR product was introduced into pCAMBIA1381 as described.

Structures of all constructs were verified by DNA sequencing. Agrobacterium tumefaciens GV3101 was transformed by electroporation, and Arabidopsis plants (Col-0) by the floral dip protocol (Clough and Bent, 1998). Seeds of the T1 generation were selected for resistance to hygromycin. Soluble extracts of T2 seeds were assayed for GUS activity exactly as described (Roschzttardtz et al., 2009). Protein concentrations were determined (Bradford, 1976), and GUS activities expressed as nanomoles of 4-methylumbelliferone per hour per milligram of protein.

\section{Transient Expression Assays}

The protocol of Yoo et al. (2007) was followed to isolate and transform protoplasts from fully expanded leaves of 6-8 weekold healthy plants grown under short-day conditions. Three types of plasmid DNA were used for protoplast transformation: a reporter plasmid with the wild type or mutated $S D H 2.3$ promoter $(-114$ to +49$)$ fused to GUS in the pBT10-GUS vector (SprengerHaussels and Weisshaar, 2000), effector plasmids expressing bZIP or B3 domain transcription factors under the control of the CaMV35S promoter in the pUCpSS vector (kindly gift of Dr. Patricio Arce) and a plasmid expressing NAN gene under the control of the CaMV35S promoter (transfection efficiency control for normalization, Kirby and Kavanagh, 2002). For each transfection, $9 \mu \mathrm{g}$ of reporter plasmid, $6 \mu \mathrm{g}$ of each effector plasmid and $3 \mu \mathrm{g}$ of the NAN plasmid were used. The overall amount of DNA was set to $30 \mu \mathrm{g}$ by adding pUCpSS plasmid and used to transfect $3 \times 10^{4}$ protoplasts in $200 \mu \mathrm{l}$. All transfections

${ }^{2}$ http://www.cambia.org 
were done in triplicate. Protoplast lysates were prepared in GUS buffer (Jefferson, 1987), and GUS and NAN enzyme assays were performed as described by Kirby and Kavanagh (2002).

To generate the reporter plasmid, the $S D H 2.3$ promoter region was amplified by PCR using Arabidopsis genomic DNA as template, forward primer sdh2.3F3 and reverse primer sdh2.3R. Mutated SDH2.3 promoter was obtained by using as template genomic DNA from transgenic plants carrying the SDH2.3 promoter mutated in the ABRE2 element (Roschzttardtz et al., 2009). PCR products were cloned into pGEM-T plasmid, and DNA fragments obtained by digestion with HindIII and NcoI were ligated into pBT10-GUS digested with the same restriction enzymes.

Effector plasmids for the expression of Arabidopsis bZIP10, bZIP25, bZIP53, and ABI3 were generated by cloning the corresponding cDNAs between the CaMV35S promoter and terminator in pUCpSS. cDNAs were obtained by RT-PCR with the following primer pairs: bzip10F and bZIP10R, bzip25F and bzip25R, bzip53F and bzip53R, and abi3F and abi3R (Supplementary Table S1). PCR products were cloned into pGEM-T, recombinant plasmids were digested with the appropriate restriction enzymes and the DNA inserts were ligated into pUCpSS.

Structures of all constructs were verified by DNA sequencing and plasmids DNAs were purified by centrifugation in $\mathrm{CsCl}$ gradients.

\section{Expression Analysis by Real-Time qRT-PCR}

The method of Oñate-Sanchez and Vicente-Carbajosa (2008) was employed to extract total RNA from $25 \mathrm{mg}$ of seeds. cDNA synthesis was carried out with $2 \mu \mathrm{g}$ of DNase I-pretreated RNA, M-MLV Reverse Transcriptase (Promega), and random hexamers as primers. To evaluate gene expression, the primer pairs were RTsdh2.3F and RTsdh2.3R for SDH2.3, and RTclnF and RTclnR for clathrin. One tenth of the cDNA was employed in qRT-PCR assays, using the Mx3000P QPCR System (Stratagene) and the SensiMix Plus SYBR kit provided by Quantace. Expression values were calculated considering the amplification efficiency of each primer pair, and were normalized using the clathrin transcript as an internal control.

\section{Succinate Dehydrogenase Activity Staining}

A simplified procedure was used, based on that described by Baud and Graham (2006). All steps, from imbibition to staining, were performed in the absence or presence of 1 or $10 \mu \mathrm{M}$ cycloheximide. Seeds were imbibed in the dark for $16 \mathrm{~h}$ at $4^{\circ} \mathrm{C}$, embryos were excised, incubated for $2 \mathrm{~h}$ at $37^{\circ} \mathrm{C}$ in $50 \mathrm{mM}$ sodium phosphate ( $\mathrm{pH} 7.6$ ), and finally stained by incubation for $1-3 \mathrm{~h}$ at $30^{\circ} \mathrm{C}$ in $50 \mathrm{mM}$ sodium phosphate $(\mathrm{pH} 7.6), 7.5 \mathrm{mM}$ succinate (freshly prepared) and $2.4 \mathrm{mM}$ nitroblue tetrazolium. In parallel, embryos were incubated in the same buffer without succinate, and show negligible background activity (Figure 4). After staining, embryos were washed with $70 \%$ ethanol, viewed in a stereomicroscope (Nikon SMZ800) and photographed with a Nikon Coolpix 4500CCD camera.

\section{Statistical Analyses}

Krustall-Wallis test followed by Dunn's test for multiple comparisons, and Mann-Whitney test were performed using GraphPad Prism, GraphPad Software (San Diego, CA, USA). Student's $t$-tests were performed using the algorithm embedded into Microsoft Excel.

\section{RESULTS}

\section{SDH2.3 Promoter Characterization}

We have previously shown that the region between -223 and +49 (numbers in relation to the transcription start site) is sufficient to confer high expression of the GUS reporter gene in mature embryos (Elorza et al., 2006; Figure 1). This region contains three ABRE elements and one RY-like enhancer element, which were shown by mutagenesis to be necessary for high SDH2.3 promoter activity (Roschzttardtz et al., 2009). In order to evaluate if a promoter fragment comprising the three ABRE and RY elements is sufficient for promoter activity, the region between -114 and -32 was fused to a minimal CaMV35S promoter containing TATA and CAAT boxes (construct -114/32/-64p35S in Figure 1A). Extracts from mature T2 seeds were used to measure GUS activity: a drastic reduction was found when compared to the $-223 /+49$ control construct, showing that the three ABRE and RY elements are necessary but not sufficient to drive high seed expression.

We interpreted that either the region upstream of the ABRE3 element $(-223$ to -114$)$, the region downstream of the RY element $(-32$ to +49$)$, or both are required for transcription in seeds. Thus, two additional constructs were assayed, one in which the region between -223 and -114 was deleted $(-114 /+49$ in Figure 1A), and the other in which the -223 to -32 fragment was fused to the CaMV35S minimal promoter (-223/-32/-64p35S in Figure 1A). Interestingly, GUS activity was not significantly affected by deletion of the promoter to $-114(-114 /+49$ in Figure 1B), indicating that all the elements necessary for high seed expression are present in the -114 to +49 region. In contrast, GUS activity was abolished almost completely when the region downstream the RY element was absent (-223/-32/-64p35S in Figure 1B). Furthermore, very weak or no GUS activity was detected when the -114 to -1 region of the SDH2.3 promoter was fused to the $5^{\prime}$ UTR of the CaMV35S promoter in pBI121 (-114/-1/35S 5'UTR in Figure 1A) (Figure 1B). Altogether, these results showed that cis-elements located between -32 and +49 are necessary for a functional $S D H 2.3$ promoter, in addition to the upstream ABRE and RY elements. Moreover, they suggest that at least some of these cis elements are located in the region encoding the $5^{\prime}$ UTR.

In silico analysis revealed the presence of two putative ciselements that may be involved in the regulation of seed gene expression (e.g., Marzabal et al., 2008; Agarwal et al., 2011) and are located downstream of the RY element. These elements, an auxin-responsive element (AuxRE) and a DOF transcription 
A

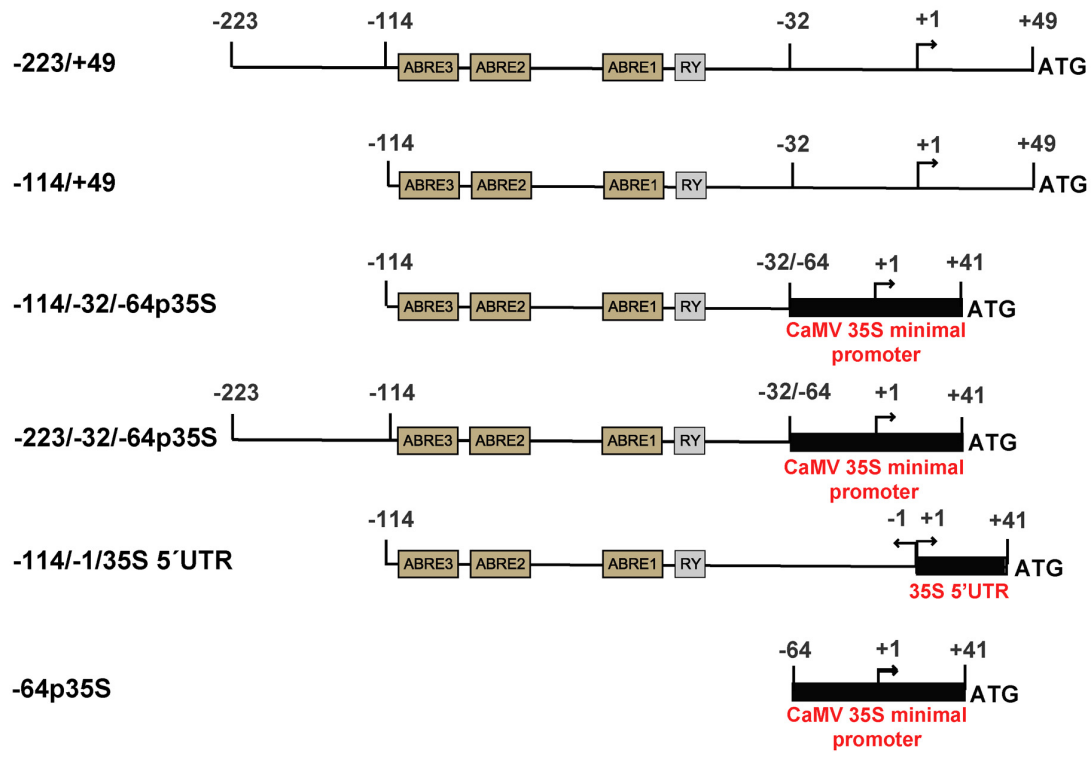

B

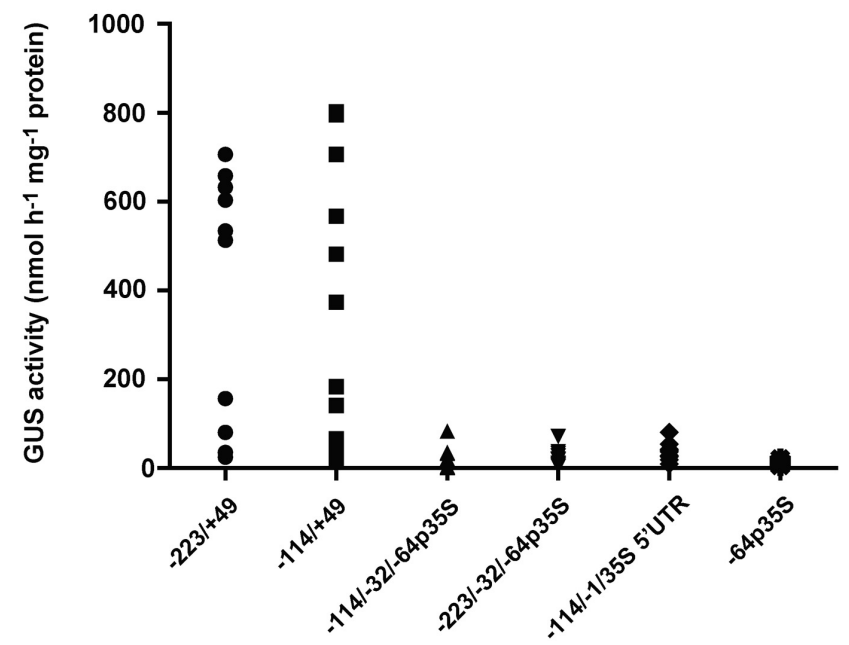

FIGURE 1 | Identification of the SDH2.3 functional promoter. (A) Structure of the constructs fused to GUS. Thin lines with motifs shown in boxes represent the $S D H 2.3$ promoter. Numbers are in relation to the transcription initiation site, indicated by a curved arrow. Black boxes represent the CaMV35S minimal promoter and the region encoding its $5^{\prime}$ UTR. (B) GUS activity was measured in duplicate seed extracts from 10, 17, 11, 11, 11, and 6 independent transgenic lines carrying the -223/+49, -114/-32/-64p35S, -114/+49. -223/-32/-64p35S, -114/-1/35S 5'UTR and -64p35S constructs fused to GUS, respectively. Each symbol represents one transgenic line.

factor-binding site (Supplementary Figure S1A) were mutated either alone or in combination, without significant changes in promoter activity (Supplementary Figure S1B). Thus, these elements play a minor role, if any, in $S D H 2.3$ promoter activity.

\section{bZIP Transcription Factors and ABA Regulate SDH2.3 Expression}

We have previously shown that transcription factors of the B3 domain family regulate $S D H 2.3$ expression in vivo (Roschzttardtz et al., 2009). In contrast, although we were able to demonstrate that the transcription factors bZIP53, bZIP10, and bZIP25 bind in vitro to the ABRE motifs in the SDH2.3 promoter, no functional data about the in vivo role in regulating $S D H 2.3$ expression was obtained. Accordingly, we first analyzed $S D H 2.3$ expression in bzip53, bzip10, and bzip25 homozygous mutant seeds (Figure 2A). We have chosen the group S1 bZIP53 (Jakoby et al., 2002) because this transcription factor has an important role in seed maturation gene expression (Alonso et al., 2009), and bZIP10 and bZIP25, two members of group $\mathrm{C}$ of bZIPs, because they are dimerizing partners of bZIP53 


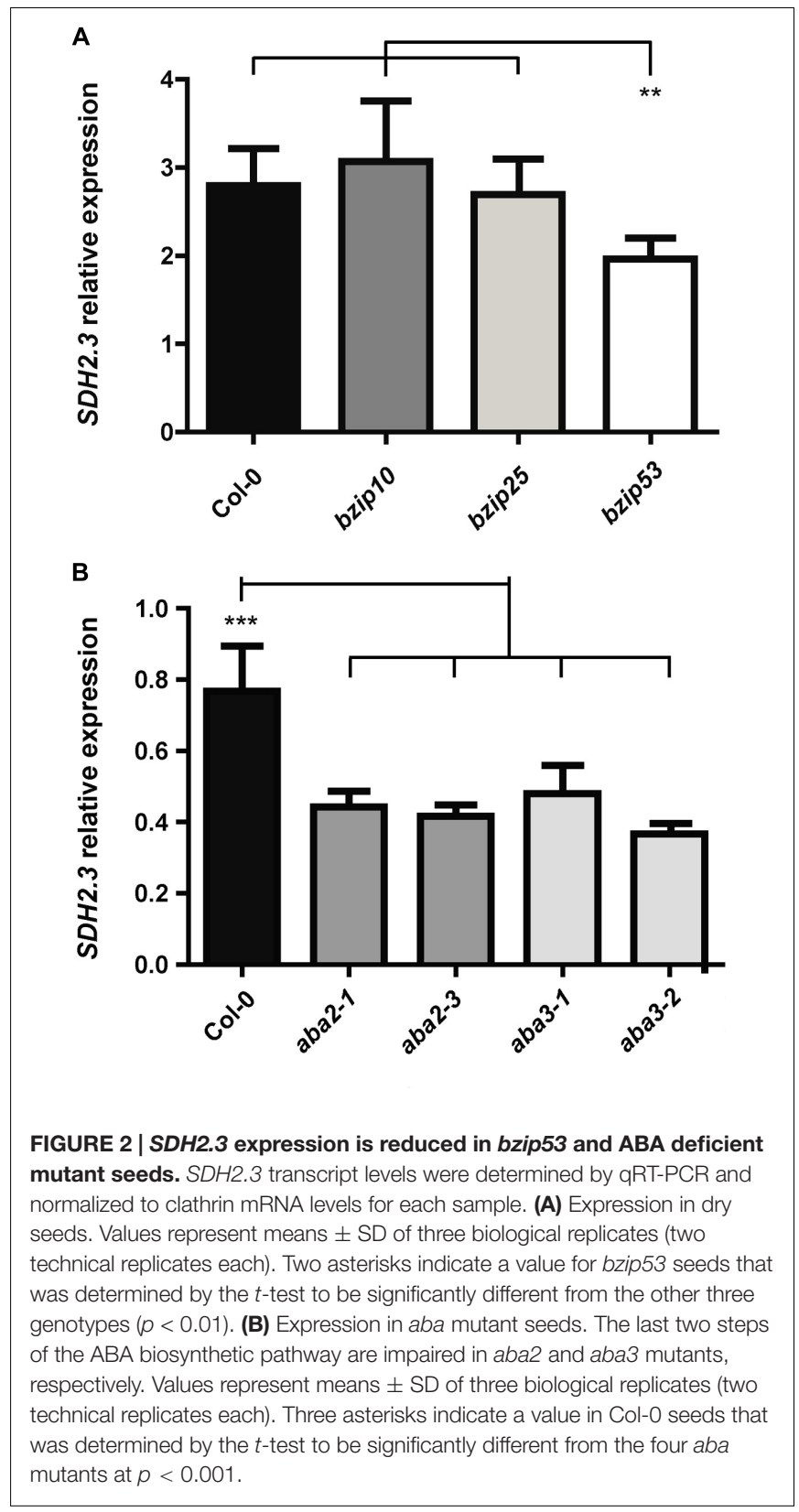

(Ehlert et al., 2006; Weltmeier et al., 2006) and have been shown to induce SSP gene expression (Lara et al., 2003; Alonso et al., 2009).

The accumulation of SDH2.3 transcripts was significantly reduced in bzip53 knockdown seeds whereas bzip10 and bzip25 mutant seeds did not show any significant difference when compared to wild type seeds (Figure 2A). These results reveal that bZIP53 is likely involved in the in vivo regulation of SDH2.3 expression. A plausible explanation for unaltered transcript levels in bzip10 and bzip25 mutant seeds may be redundancy between these transcription factors and/or with other bZIPs from the $\mathrm{C}$ group.

In a second approach, we performed transient expression analysis in leaf protoplasts using the $S D H 2.3$ promoter

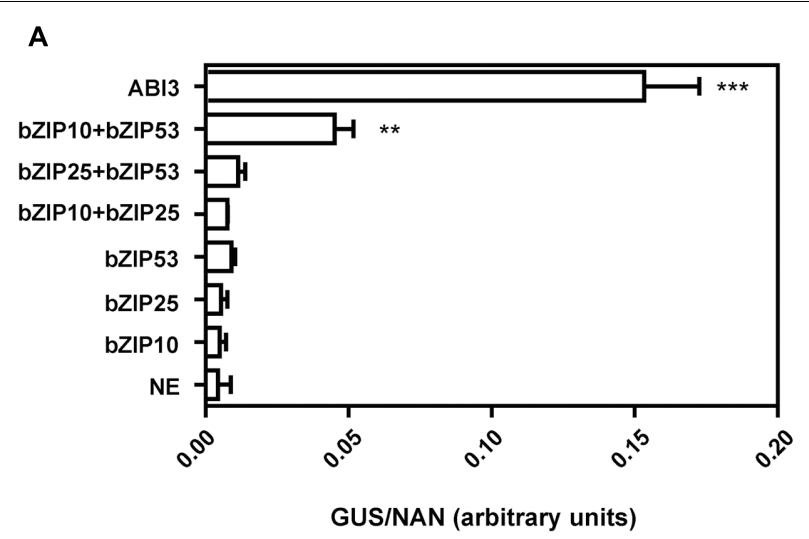

B

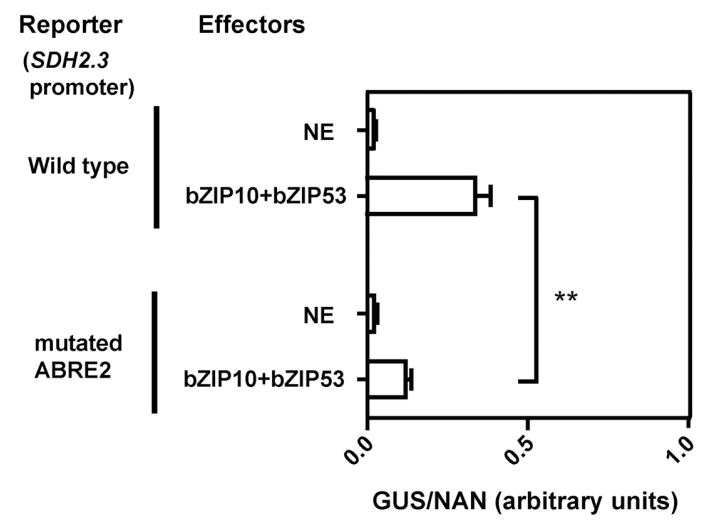

FIGURE 3 | bZIP53 and bZIP10 transcription factors activate the SDH2.3 promoter. (A) Arabidopsis protoplasts were transfected with a reporter construct (SDH2.3 promoter fused to GUS) and effector constructs for expression of either bZIP or ABI3 transcription factors under the control of the CaMV35S promoter. A control plasmid expressing NAN was included in all transfections to normalize GUS activities for differences in transfection efficiencies. $x$-axis values are given as GUS activity to NAN activity (arbitrary units). NE is no effector(s) (replaced by the pUCpSS vector). Values are means $\pm S D$ of three replicates. Asterisks indicate values that were determined by the $t$-test to be significantly different from that in NE $(* * p<0.01 ; * * * p<0.001)$. All other GUS/NAN values are not significantly different form that in NE. (B) Arabidopsis protoplasts were transfected with either the same reporter as in (A) (wild type SDH2.3 promoter fused to GUS) or a reporter construct with a mutated version of the $S D H 2.3$ promoter, in which a modified ABRE2 element was fused to GUS. Effector constructs expressed bZIP transcription factors. Values are means \pm SD of three replicates. Promoter activities in the presence of bZIP53+bZIP10 are significantly different from activities in NE $(p<0.001$, asterisks not shown). Two asterisks indicate a significant difference at $p<0.01$.

$(-114$ to +49$)$ to drive GUS expression. This reporter plasmid was cotransfected with effector plasmids expressing bZIP and/or the $\mathrm{B} 3$ domain transcription factor $\mathrm{ABI} 3$, and a plasmid expressing NAN to normalize for transfection efficiency. Individually bZIP10, bZIP25, and bZIP53 were unable to activate the SDH2.3 promoter (Figure 3A). Only when bZIP53 and bZIP10 were cotransfected a significant increase in promoter activity was observed, showing for the first time that bZIP transcription factors directly activate the $S D H 2.3$ promoter. When a modified version of the SDH2.3 promoter was used 
in which the ABRE2 element was mutated to a sequence that results in a drastic reduction of in planta promoter activity (Roschzttardtz et al., 2009), the activation mediated by bZIP53+bZIP10 was significantly reduced, indicating that the regulation of the $S D H 2.3$ promoter by these bZIP factors requires intact $\mathrm{ABRE}$ motifs (Figure 3B). In our transient assays $\mathrm{ABI} 3$ was able to activate the $S D H 2.3$ promoter even more efficiently than bZIP53+bZIP10 (Figure 3A), and the inclusion of bZIP transcription factors did not produce any additional increase in promoter activation (Supplementary Figure S2). These results confirm our previous data suggesting a direct effect of $\mathrm{ABI} 3$ on the SDH2.3 promoter.

ABRE elements are bound by bZIP transcription factors and are supposed to mediate ABA effects on transcription. To evaluate if ABA is indeed involved in the regulation of $S D H 2.3$ expression in seeds, i.e., where $S D H 2.3$ is expressed in vivo, SDH2.3 transcript levels were measured in wild type and ABA deficient seeds (Figure 2B). The level of SDH2.3 mRNA was significantly lower in seeds from mutants impaired in the last two steps of the ABA synthesis pathway, revealing that $\mathrm{ABA}$ is involved in the in vivo regulation of seed $S D H 2.3$ expression.

Altogether, our results show that a -114 to +49 promoter region is necessary and sufficient for seed specific expression of the SDH2.3 gene, encoding an iron-sulfur subunit of mitochondrial complex II. ABI3, bZIP10/bZIP53 heterodimers, and the hormone ABA regulate $S D H 2.3$ expression.

\section{A Complex II Containing SDH2.3 as Iron-Sulfur Subunit Is Present in Mature Embryos Before Imbibition}

The SDH2.3 transcript level is high in dry seeds (Elorza et al., 2006). To evaluate if, before imbibition and germination, the SDH2.3 polypeptide is translated and already present in mature embryos and $\mathrm{SDH}$ is active, wild type seeds and seeds from single sdh2.3 mutants, single sdh2.1 mutants and double $s d h 2.3 / s d h 2.1$ mutants were imbibed in the presence (or absence) of the protein synthesis inhibitor cycloheximide. Then all steps, i.e., embryo excision and staining for SDH activity, were performed in the presence (or absence) of cycloheximide. SDH activity was clearly detected in wild type embryos and, most importantly, is insensitive to 1 or $10 \mu \mathrm{M}$ cycloheximide (Figure 4, middle and lower panels). Evidence for effective entry of cycloheximide into the embryo is given by complete inhibition of germination (Supplementary Figure S3). SDH activity was apparently unaffected in sdh2.1 single mutants (Figure 4, upper panel) and was greatly reduced in both $s d h 2.3$ knockout mutants (Figure 4, middle panel). Thus, respiratory Complex II is already present in mature seeds before imbibition and contains mainly SDH2.3 as iron-sulfur subunit. However, SDH activity was detected in sdh2.3 single mutants, and this activity results from basal expression of SDH2.1, since double $s d h 2.3 / s d h 2.1$ mutants lack any visible staining (Figure 4, lower panel). Semiquantitative estimation of staining performed as described by Baud and Graham (2006) suggests that single sdh2.3 mutants have $5-10 \%$ of the wild type SDH activity, and that double $s d h 2.3 / s d h 2.1$ show negligible activity.

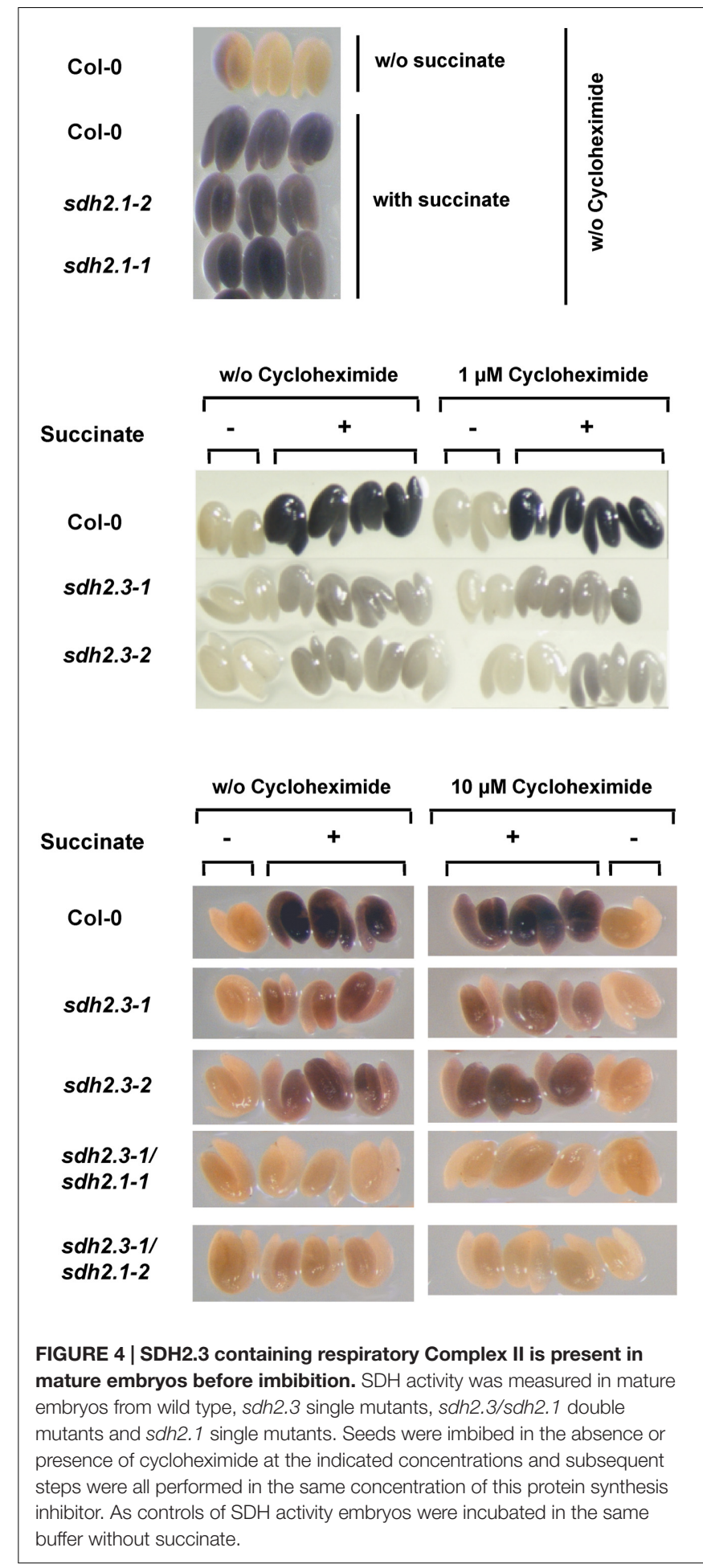

\section{Complex II Plays a Role in Germination and is Essential for Seedling Establishment}

Germination sensu-stricto (radicle protrusion) of sdh2.3 mutant seeds were delayed when compared to that of wild type 
seeds, however, all seeds germinated and seedlings established (Roschzttardtz et al., 2009). To further analyze the role of Complex II during germination and early post-germinative growth, low concentrations of TTFA, a known specific noncompetitive Complex II inhibitor binding at the ubiquinone pocket (Sun et al., 2005), were shown to be able to delay germination without blocking it (Figure 5A). When germination was evaluated at 10 days it was only slightly inhibited by
TTFA (Figure 5B), and at concentrations higher than $100 \mu \mathrm{M}$ (e.g., $0.25 \mathrm{mM}$ TTFA inhibits germination by only $22 \%$ ). Thus, a SDH2.3 containing Complex II appears to play an important but not essential role at very early stages of plant development. By comparison, germination is more sensitive to sodium azide, a cytochrome c oxidase (complex IV) inhibitor, being completely blocked at $0.1 \mathrm{mM}$ (Supplementary Figure S4).

\section{A}

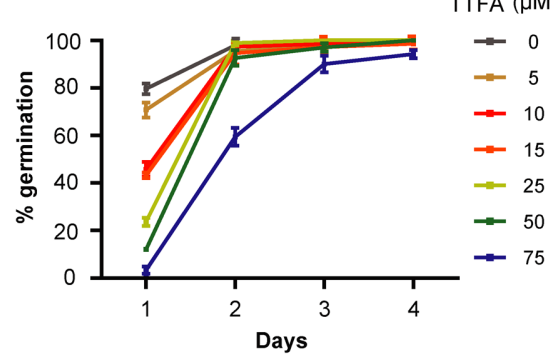

C

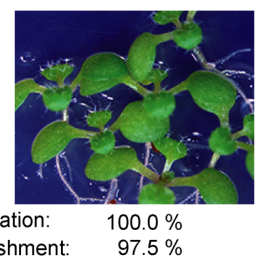

Establishment: $\quad 97.5 \%$

$50 \mu \mathrm{M}$ TTFA

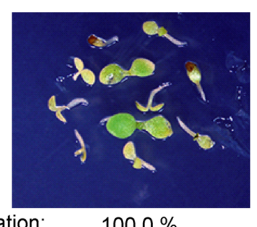

Germination: $\quad 100.0 \%$

Establishment:

$100 \mu \mathrm{M}$ TTFA

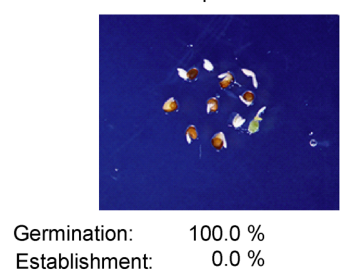

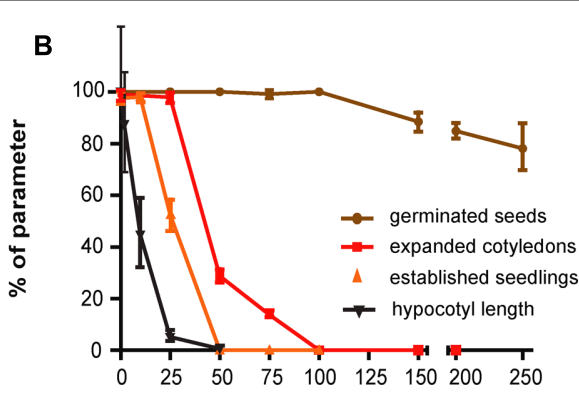

TTFA ( $\mu$ M)
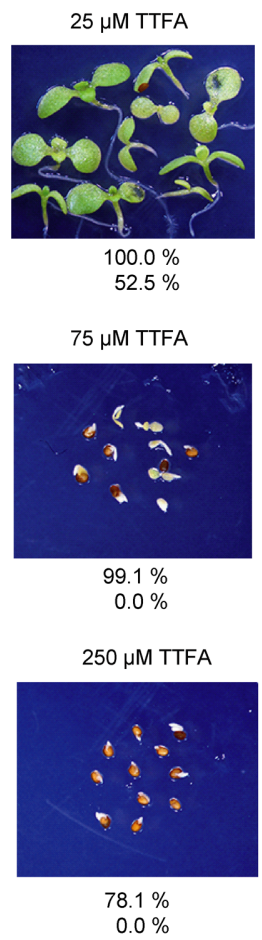

FIGURE 5 | (A) Germination is retarded by low TTFA concentrations. Wild type seeds were sown on half-strength MS agar plates without TTFA or with varying concentrations of this complex II inhibitor. Germination was scored at different times and is complete at 4 days. Values are means $\pm S D$ of three replicates, each containing 80-200 seeds. (B,C) Post-germinative growth is strongly inhibited by TTFA. Seeds were sown on half-strength MS agar plates, stratified, and grown for 10 days under long-day conditions to evaluate germination (brown symbols), cotyledon expansion (red symbols) and seedling establishment (orange symbols). In a different experiment, they were grown in the dark to measure hypocotyl elongation (black symbols). Values are means \pm SD of three replicates each containing around 50 sown seeds. Germinated seeds, seedlings with expanded cotyledons and seedlings with true leaves were counted, and values are given as percentages of sown seeds. Hypocotyl lengths are given as percentages of hypocotyl length in the absence of TTFA. (C) Photographs were taken at the same magnification after 10 days of growth under long-day conditions. 
In contrast to the moderate effect of TTFA on germination, cotyledon expansion (and greening), hypocotyl elongation and seedling establishment are more sensitive to TTFA (Figure 5). For instance, seedling establishment is completely blocked by 50-75 $\mu \mathrm{M}$ TTFA (Figure 5B) and seedlings remain white (Figure 5C). Similar results were obtained with two additional Complex II inhibitors: carboxin (Figure 6), a non-competitive inhibitor binding at the quinone site, and malonate (Figure 7), a competitive inhibitor at the succinate binding site. Importantly, double $s d h 2.3 / s d h 2.1$ mutants are more sensitive to these inhibitors (Figures 6, 7), strongly suggesting that seedling greening and establishment is blocked through Complex II inhibition. Altogether, these results suggest that Complex II is essential for chloroplast biogenesis, the acquisition of photosynthetic competence and ultimately seedling establishment.

\section{DISCUSSION}

\section{Regulation of SDH2.3 Expression}

The SDH2.3 gene, encoding an isoform of the Complex II ironsulfur subunit, is the only described TCA cycle and ETC gene to be specifically expressed in the embryo during seed maturation, and this expression is controlled at transcription (Elorza et al., 2006; Roschzttardtz et al., 2009). We have now defined the region
$(-114$ to +49$)$ that contains all the cis-elements needed for high seed transcription (Figure 1). This region includes between -114 and -32 three ABRE elements and one RY element which are necessary but not sufficient for seed expression. Thus, further elements located between -31 and +49 are required, and at least some of them appear to be present in the region encoding the $5^{\prime}$ UTR since exchanging the $S D H 2.35^{\prime}$ UTR for a known functional $5^{\prime}$ UTR inactivates the promoter (Figure 1, construct -114/-1/35S $5^{\prime}$ UTR). It is likely that this region $(-31$ to +49$)$ contains the core promoter elements needed to assemble the transcription pre-initiation complex. Unfortunately, these elements are not well-characterized in plants, particularly in promoters such as the SDH2.3 promoter that lack a TATA box (e.g., Molina and Grotewold, 2005; Morton et al., 2014).

Group S1 bZIP53 has an important role in seed maturation gene expression (Alonso et al., 2009), and bZIP10 and bZIP25 have been shown to activate SSP gene transcription synergistically with ABI3 (Lara et al., 2003). Here we demonstrate that bZIP transcription factors activate the $S D H 2.3$ promoter and regulate SDH2.3 expression in vivo. First, we found that SDH2.3 mRNA levels are reduced in a mutant with lowered bZIP53 expression (bzip53 knockdown mutant, Figure 2A). And second, bZIP53+bZIP10 activate the SDH2.3 promoter in leaf protoplasts, and this activation is reduced when the ABRE2 cis-element is mutated to prevent transcription factor binding (Figure 3). In these transient assays, individual bZIP
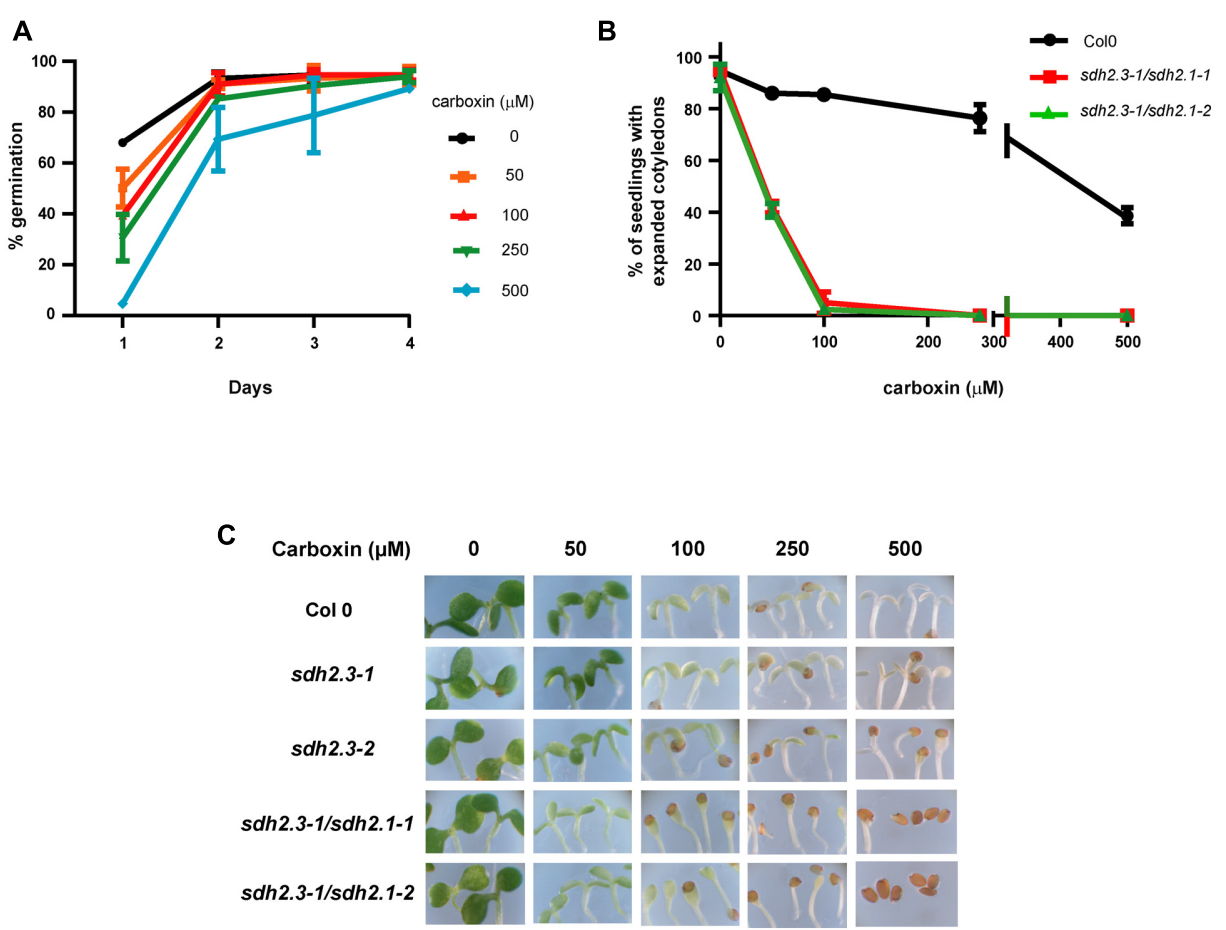

FIGURE 6 | (A) Germination is retarded by carboxin. Wild type seeds were sown on half-strength MS agar plates without carboxin or with varying concentrations of this complex II inhibitor. Germination was scored at different times. Values are means \pm SD of three replicates, each containing 50-90 seeds. (B,C) Post-germinative growth of double sdh2.3/sdh2.1 mutants is more sensitive to carboxin inhibition. Seeds were sown on half-strength MS agar plates supplemented with different concentrations of carboxin, stratified, and grown under long-day conditions. (B) Cotyledon expansion was recorded at 5 days and values are means \pm SD (each replicate containing 40-90 seeds). (C) Photographs were taken after 8 days of growth. 

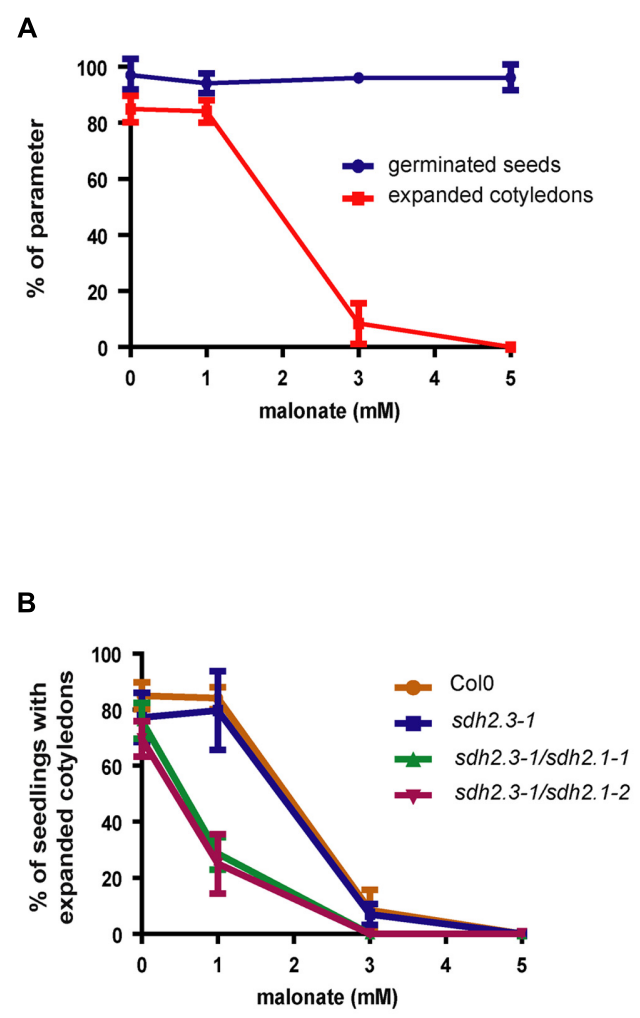

C

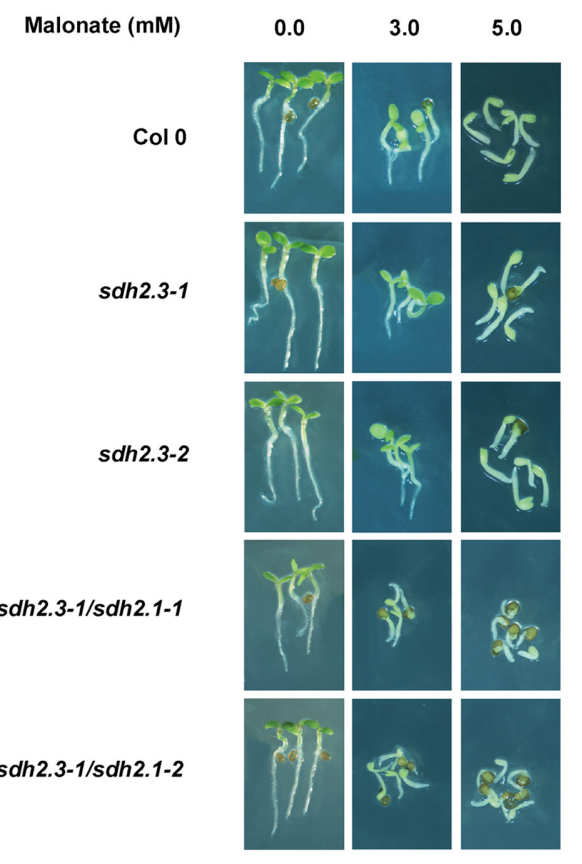

FIGURE 7 | (A) Post-germinative growth is inhibited by malonate. Wild type seeds were sown on half-strength MS agar plates without malonate or with varying concentrations of this complex II inhibitor, stratified and grown under long-day conditions. Germination (blue symbols) and cotyledon expansion (red symbols) were evaluated at $30 \mathrm{~h}$ and 3 days, respectively. Values are means \pm SD of three replicates, each containing 30-50 seeds. (B,C) Post-germinative growth of double sdh2.3/sdh2.1 mutants are more sensitive to malonate inhibition. Seeds were sown, stratified, and grown under long-day conditions. (B) Cotyledon expansion was evaluated for each genotype at 3 days, and values are means \pm SD of three replicates, each containing 30-60 seeds. (C) Photographs were taken after 3 days of growth.

transcription factors and, interestingly, the bZIP53+bZIP25 combination were unable to activate the SDH2.3 promoter. This apparent specificity for bZIP10 may be related to the fact that bZIP53/bZIP10 heterodimers bind better in vitro to the promoter than bZIP53/bZIP25 (Roschzttardtz et al., 2009). However, we cannot exclude a role for bZIP25 in SDH2.3 regulation during embryo maturation since it is closely related to bZIP10, they are the group C bZIPs with higher expression in developing seeds (Lara et al., 2003), and non-seed models such as mesophyll protoplasts might not reflect the native situation in seeds. Given that bZIP53 has been identified as the major group S1 bZIP member expressed during seed maturation (Alonso et al., 2009), our results provide strong evidence that the SDH2.3 gene is a direct target of bZIP53 transcription factor, likely as bZIP53/bZIP10 heterodimers. In addition to its role in the regulation of a respiratory complex subunit (this work) and SSP genes during seed maturation (Alonso et al., 2009), bZIP53 is involved in the regulation of amino acid metabolism during low energy and salt/osmolarity stresses in adult plants (Weltmeier et al., 2006; Dietrich et al., 2011; Hartmann et al., 2015). The results from transient expression assays also confirm our previous data on the direct role of $\mathrm{ABI} 3$ in the regulation of $S D H 2.3$ transcription (Figure 3A).
Finally, we have demonstrated that the hormone ABA, known for its role in seed maturation and acquisition of desiccation tolerance, is involved in the regulation of $S D H 2.3$ expression in seeds (Figure 2B). Nevertheless, SDH2.3 expression is not induced in vegetative tissues by either ABA or saline and osmotic stresses (results not shown). Similar results have been reported for SSP genes and are likely due to the lack of the seed-specific B3 domain transcription factors. Regulation of the mitochondrial ETC by ABA is not without precedent, although only in vegetative tissue. Indeed, expression of the gene encoding Alternative oxidase 1a is repressed by ABI4 (ABA-insensitive 4) transcription factor and induced by ABA (Giraud et al., 2009), and $A B A$ reduced the expression of a mitochondrial DEXH box RNA helicase involved in splicing of transcripts encoding complex I subunits (He et al., 2012).

In summary, the $S D H 2.3$ promoter appears to be regulated in a similar way to that of SSP genes (e.g., 2 S albumins): they are induced early in maturation, they have similar cis-elements, they are the targets of bZIP and B3 domain transcription factors. However, some differences exist: whereas expression of SSP genes decreases during desiccation, SDH2.3 mRNA remains high. These differences may be due to different promoter architectures and their interaction with transcription factors. 


\section{Complex II Role during Germination and Establishment}

In our previous work, we have shown that SDH2.3 transcripts accumulate in maturing and dry embryos (Elorza et al., 2006). Here we conclusively demonstrated, by using a protein synthesis inhibitor during imbibition and SDH activity assays, that a SDH2.3 containing Complex II is already present in the embryo, before germination (Figure 4). Furthermore, the use of single $s d h 2.3$ and $s d h 2.1$ mutants, and double $s d h 2.3 / s d h 2.1$ mutants allowed us to conclude that most Complex II $(\sim 90 \%)$ in mature embryos contains $\mathrm{SDH} 2.3$ as the iron-sulfur subunit, a little fraction $(\sim 10 \%)$ contains SDH2.1, and SDH2.2 is absent. These results are consistent with the previously reported weak expression of a fusion between the SDH2.1 promoter to GUS in maturing embryos, and the lack of GUS expression with a similar construct for the $S D H 2.2$ gene (Roschzttardtz et al., 2009).

Germination sensu-stricto (until radicle emergence) is shortly followed by seed reserve mobilization which fuel growth until the seedling becomes photoautotrophic, both steps being crucial for seedling establishment (Bewley, 1997; Eastmond and Graham, 2001). In different plant species, including Arabidopsis, seed metabolic activity resumes within minutes of imbibition, and respiration through the standard ETC rapidly increases at the earliest stages of germination (e.g., Ehrenshaft and Brambl, 1990; Benamar et al., 2003; Howell et al., 2006; Sew et al., 2013). Moreover, germination of maize (Ehrenshaft and Brambl, 1990), pea (Benamar et al., 2003), and Arabidopsis (Supplementary Figure S4) seeds is dependent on a functional ETC, and mitochondria in dry seeds from maize, rice, and pea rapidly become active during imbibition, relying on succinate and external NADH as substrates (Ehrenshaft and Brambl, 1990; Logan et al., 2001; Benamar et al., 2003; Howell et al., 2006). In sunflower, even mitochondria extracted from dry seeds are capable of succinate-dependent $\mathrm{O}_{2}$ consumption and ATP synthesis (Attuci et al., 1991).

These observations are consistent with an active SDH2.3 containing complex II already present in mature Arabidopsis embryos (Figure 4) and suggest a role for this complex during germination sensu-stricto. This role is further supported by the delay in germination observed at low TTFA concentrations (Figure 5A) and in mutants lacking SDH2.3 (Roschzttardtz et al., 2009). However, a fully active complex II, although contributing to an efficient germination, is not essential at this early step, and this may be explained by mitochondrial oxidation of other substrates, in particular external NADH as mentioned above. The resumption of respiratory activity depending upon a respiratory system present in the dry embryo and able to oxidize succinate and external NADH and to synthesize ATP very early after imbibition, will be a prerequisite for later mitochondrial biogenesis involving gene expression and import of ETC and TCA cycle components, among others (Logan et al., 2001; Howell et al., 2006; Narsai et al., 2011; Weitbrecht et al., 2011).

At present, we can only speculate about the selective advantage that SDH2.3 may confer to complex II. It is interesting to note that transcripts from all other SDH canonical subunits are present in dry seeds and their levels do not change significantly until $24 \mathrm{~h}$ post-imbibition (Supplementary Table S1 in Weitbrecht et al., 2011). A SDH2.1/SDH2.2 like gene has been described in maize, rice and wheat (Kubo et al., 1999; Sandoval et al., 2004), and in databases we have identified a SDH2.3 homolog in several monocots and dicots (unpublished results). Thus, the presence of two kinds of $\mathrm{SDH} 2$ proteins seems conserved among angiosperms, and a specific embryo iron-sulfur subunit may have evolved to deal with the stressful conditions encountered during desiccation (e.g., to protect ironsulfur centers from oxidative damage).

Beyond its role in germination, our results indicate that complex II has an essential function during early postgerminative growth, for the acquisition of photosynthetic competence and seedling establishment (Figures 5-7). Single $s d h 2.3$ and double $s d h 2.3 / s d h 2.1$ mutants are able to switch to photoautotrophism and develop normally in the light (e.g., Figures 6, 7), however, interpretation of these results in terms of complex II function is complicated by the fact that $S D H 2.2$ and SDH2.1 are rapidly induced after imbibition $(3 \mathrm{~h})$, at least at the transcript level (Weitbrecht et al., 2011), and double $s d h 2.1 / s d h 2.2$ or triple $s d h 2.1 / s d h 2.2 / s d h 2.3$ are not viable. For these reasons, we use complex II inhibitors and found that they block hypocotyl elongation in the dark and the switch from heterotrophic to autotrophic growth in the light. Interestingly, recent studies have shown that complex $I$ is also required for seedling establishment (Kühn et al., 2015), suggesting a general role for the mitochondrial ETC in providing energy for this crucial developmental step.

After germination, growth and seedling establishment depends largely on fatty acid oxidation. Glyoxysomal $\beta$-oxidation converts fatty acids to acetyl-CoA, which is subsequently used to produce succinate via the glyoxylate cycle (Eastmond and Graham, 2001; Graham, 2008). Succinate is then transported to mitochondria, where it can be used as a respiratory substrate or as a gluconeogenic substrate, and in both cases complex II activity would be necessary. Thus, complete complex II inhibition would impair both ATP production and the synthesis of sucrose required to fuel growth of new tissues. Although the precise mechanisms which lead to the failure in chloroplast development are unknown, the link between mitochondrial function and chloroplast development is not without precedent. Indeed, there are a number of non-chromosomal stripe (NCS) mutants in maize that carry deletions in essential mitochondrial genes and are maintained heteroplasmically (e.g., Roussell et al., 1991; Gu et al., 1993). These plants exhibit yellow or pale green stripes on the leaves which result from defective chloroplasts in sectors carrying the mitochondrial mutations, supporting the requirement of mitochondrial function for the development of photosynthetically active chloroplasts.

Few mutants in SDH subunits or assembly factors have been characterized (reviewed in Huang and Millar, 2013). Surprisingly, these studies have revealed complex II roles in stomatal function (Araújo et al., 2011; Fuentes et al., 2011), root elongation (Huang et al., 2013), plant defense to pathogens through ROS generation (Gleason et al., 2011), and pollen and ovule development (León et al., 2007). We have now provided evidence for roles in 
seed germination and seedling establishment. Future research would be necessary to understand at the tissue-specific level the mechanisms operating to explain this diversity in "the complex roles of this simple enzyme" (Huang and Millar, 2013).

\section{AUTHOR CONTRIBUTIONS}

FR, JV-C, and XJ design the research. FR, RE-C, and IG carried out the experiments and analyzed the data. FR, RE-C, JV-C, and $\mathrm{XJ}$ contributed to writing the manuscript. XJ and JV-C supervised the project.

\section{REFERENCES}

Agarwal, P., Kapoor, S., and Tyagi, A. K. (2011). Transcription factors regulating the progression of monocot and dicot seed development. Bioessays 33, 189-202. doi: 10.1002/bies.201000107

Alonso, R., Oñate-Sanchez, L., Weltmeier, F., Ehlert, A., Diaz, I., Dietrich, K., et al. (2009). A pivotal role of the basic leucine zipper transcription factor bZIP53 in the regulation of Arabidopsis seed maturation gene expression based on heterodimerization and protein complex formation. Plant Cell 21, 1747-1761. doi: $10.1105 /$ tpc. 108.062968

Araújo, W. L., Nunes-Nesi, A., Osorio, S., Usadel, B., Fuentes, D., Nagy, R., et al. (2011). Antisense inhibition of the iron-sulphur subunit of succinate dehydrogenase enhances photosynthesis and growth in tomato via an organicacid-mediated effect on stomatal aperture. Plant Cell 23, 600-627. doi: 10.1105/ tpc. 110.081224

Attuci, S., Carde, J. P., Raymond, P., Saint-Gès, V., Spiteri, A., and Pradet, A. (1991). Oxidative phosphorylation by mitochondria extracted from dry sunflower seeds. Plant Physiol. 95, 390-398. doi: 10.1104/pp.95.2.390

Barrero, J. M., Rodriguez, P. L., Quesada, V., Piqueras, P., Ponce, M. R., and Micol, J. L. (2006). Both abscisic acid (ABA)-dependent and ABA-independent pathways govern the induction of NCED3, AAO3 and ABA1 in response to salt stress. Plant Cell Environ. 29, 2000-2008. doi: 10.1111/j.1365-3040.2006. 01576.x

Baud, S., and Graham, I. A. (2006). A spatiotemporal analysis of enzymatic activities associated with carbon metabolism in wild-type and mutant embryos of Arabidopsis using in situ histochemistry. Plant J. 46, 155-169. doi: 10.1111/j. 1365-313X.2006.02682.x

Benamar, A., Tallon, C., and Macherel, D. (2003). Membrane integrity and oxidative properties of mitochondria isolated from imbibing pea seeds after priming or accelerated ageing. Seed Sci. Res. 13, 35-45. doi: 10.1079/ SSR2002122

Bewley, J. D. (1997). Seed germination and dormancy. Plant Cell 9, 1055-1066. doi: $10.1105 /$ tpc.9.7.1055

Bradford, M. M. (1976). A rapid and sensitive method for the quantitation of microgram quantities of protein utilizing the principle of protein-dye binding. Anal. Biochem. 72, 248-254. doi: 10.1016/0003-2697(76)90527-3

Busk, P. K., and Pagès, M. (1998). Regulation of abscisic acid-induced transcription. Plant Mol. Biol. 37, 425-435. doi: 10.1023/A:1006058700720

Clough, S. J., and Bent, A. F. (1998). Floral dip: a simplified method for Agrobacterium-mediated transformation of Arabidopsis thaliana. Plant J. 16, 735-743. doi: 10.1046/j.1365-313x.1998.00343.x

Dietrich, K., Weltmeier, F., Ehlert, A., Weiste, C., Stahl, M., Harter, K., et al. (2011). Heterodimers of the Arabidopsis transcription factors bZIP1 and bZIP53 reprogram amino acid metabolism during low energy stress. Plant Cell 23, 381-395. doi: 10.1105/tpc.110.075390

Eastmond, P. J., and Graham, I. A. (2001). Re-examining the role of the glyoxylate cycle in oilseeds. Trends Plant Sci. 6, 72-78. doi: 10.1016/S1360-1385(00) 01835-5

Ehlert, A., Weltmeier, F., Wang, X., Mayer, C. S., Smeekens, S., VicenteCarbajosa, J., et al. (2006). Two-hybrid protein-protein interaction analysis in Arabidopsis protoplasts: establishment of a heterodimerization map of group

\section{ACKNOWLEDGMENT}

This work was supported by FONDECYT-Chile (grant 1141197) and Millenium Nucleus for Plant Systems and Synthetic Biology (NC130030, Millennium Scientific Initiative, Ministerio de Economía, Chile).

\section{SUPPLEMENTARY MATERIAL}

The Supplementary Material for this article can be found online at: http://journal.frontiersin.org/article/10.3389/fpls.2017.00277/ full\#supplementary-material

C and group S bZIP transcription factors. Plant J. 46, 890-900. doi: 10.1111/j. 1365-313X.2006.02731.x

Ehrenshaft, M., and Brambl, R. (1990). Respiration and mitochondrial biogenesis in germinating embryos of maize. Plant Physiol. 93, 295-304. doi: 10.1104/pp. 93.1.295

Elorza, A., León, G., Gómez, I., Mouras, A., Holuigue, L., Araya, A., et al. (2004). Nuclear SDH2-1 and SDH2-2 genes, encoding the iron-sulfur subunit of mitochondrial complex II in Arabidopsis, have distinct cell-specific expression patterns and promoter activities. Plant Physiol. 136, 4072-4087. doi: 10.1104/ pp.104.049528

Elorza, A., Roschzttardtz, H., Gómez, I., Mouras, A., Holuigue, L., Araya, A., et al. (2006). A nuclear gene for the iron-sulfur subunit of mitochondrial complex II is specifically expressed during Arabidopsis seed development and germination. Plant Cell Physiol. 47, 14-21. doi: 10.1093/pcp/pci218

Figueroa, P., León, G., Elorza, A., Holuigue, L., Araya, A., and Jordana, X. (2002). The four subunits of mitochondrial respiratory complex II are encoded by multiple nuclear genes and targeted to mitochondria in Arabidopsis thaliana. Plant Mol. Biol. 50, 725-734. doi: 10.1023/A:1019926301981

Figueroa, P., León, G., Elorza, A., Holuigue, L., and Jordana, X. (2001). Three different genes encode the iron-sulfur subunit of succinate dehydrogenase in Arabidopsis thaliana. Plant Mol. Biol. 46, 241-250. doi: 10.1023/A: 1010612506070

Fuentes, D., Meneses, M., Nunes-Nesi, A., Araújo, W. L., Tapia, R., Gómez, I., et al. (2011). A deficiency in the flavoprotein of Arabidopsis mitocondrial complex II results in elevated photosynthesis and better growth in nitrogenlimiting conditions. Plant Physiol. 157, 1114-1127. doi: 10.1104/pp.111. 183939

Giraud, E., Van Aken, O., Ho, L. H. M., and Whelan, J. (2009). The transcription factor ABI4 is a regulator of mitochondrial retrograde expression of ALTERNATIVE OXIDASE1a. Plant Physiol. 150, 1286-1296. doi: 10.1104/ pp.109.139782

Gleason, C., Huang, S., Thatcher, L. F., Foley, R. C., Anderson, C. R., Carroll, A. J., et al. (2011). Mitochondrial complex II has a key role in mitochondrial-derived reactive oxygen species influence on plant stress gene regulation and defense. Proc. Natl. Acad. Sci. U.S.A. 108, 10768-10773. doi: 10.1073/pnas.1016060108

Graham, I. A. (2008). Seed storage oil mobilization. Annu. Rev. Plant Biol. 59, 115-142. doi: 10.1146/annurev.arplant.59.032607.092938

Gu, J., Miles, D., and Newton, K. J. (1993). Analysis of leaf sectors in the NCS6 mitochondrial mutant of maize. Plant Cell 5, 963-971. doi: 10.1105/tpc.5.8.963

Hartmann, L., Pedrotti, L., Weiste, C., Fekete, A., Schierstaedt, J., Göttler, J., et al. (2015). Crosstalk between two bZIP signaling pathways orchestrates salt-induced metabolic reprogramming in Arabidopsis roots. Plant Cell 27, 2244-2260. doi: 10.1105/tpc.15.00163

He, J., Duan, Y., Hua, G., Fan, G., Wang, L., Liu, Y., et al. (2012). DEXH box RNA helicase-mediated mitochondrial reactive oxygen species production in Arabidopsis mediates crosstalk between abscisic acid and auxin signaling. Plant Cell 24, 1815-1833. doi: 10.1105/tpc.112.098707

Howell, K. A., Millar, A. H., and Whelan, J. (2006). Ordered assembly of mitochondria during rice germination begins with promitochondrial structures rich in components of the protein import apparatus. Plant Mol. Biol. 60, 201-223. doi: 10.1007/s11103-005-3688-7 
Huang, S., and Millar, A. H. (2013). Succinate dehydrogenase: the complex roles of a simple enzyme. Curr. Opin. Plant Biol. 16, 344-349. doi: 10.1016/j.pbi.2013. 02.007

Huang, S., Taylor, N. L., Ströher, E., Fenske, R., and Millar, A. H. (2013). Succinate dehydrogenase assembly factor 2 is needed for assembly and activity of mitochondrial complex II and for normal root elongation in Arabidopsis. Plant J. 73, 429-441. doi: 10.1111/tpj.12041

Jakoby, M., Weisshaar, B., Dröge-Laser, W., Vicente-Carbajosa, J., Tiedemann, J., Kroj, T., et al. (2002). bZIP transcription factors in Arabidopsis. Trends Plant Sci. 7, 106-111. doi: 10.1016/S1360-1385(01)02223-3

Jefferson, R. A. (1987). Assaying chimeric genes in plants: the GUS gene fusion system. Plant Mol. Biol. Rep. 5, 387-405. doi: 10.1007/BF02667740

Kirby, J., and Kavanagh, T. A. (2002). NAN fusions: a synthetic sialidase reporter gene as a sensitive and versatile partner for GUS. Plant J. 32, 391-400. doi: 10.1046/j.1365-313X.2002.01422.x

Kubo, N., Harada, K., Hirai, A., and Kadowaki, K. I. (1999). A single nuclear transcript encoding mitochondrial RPS14 and SDHB of rice is processed by alternative splicing: common use of the same mitochondrial targeting signal for different proteins. Proc. Natl. Acad. Sci. U.S.A. 96, 9207-9211. doi: 10.1073/ pnas.96.16.9207

Kühn, K., Obata, T., Feher, K., Bock, R., Fernie, A. R., and Meyer, E. H. (2015). Complete mitochondrial complex I deficiency induces an up-regulation of respiratory fluxes that is abolished by traces of functional complex I. Plant Physiol. 168, 1537-1549. doi: 10.1104/pp.15.00589

Lara, P., Oñate-Sanchez, L., Abraham, Z., Ferrandiz, C., Diaz, I., Carbonero, P., et al. (2003). Synergistic activation of seed storage protein gene expression in Arabidopsis by ABI3 and two bZIPs related to OPAQUE2. J. Biol. Chem. 278, 21003-21011. doi: 10.1074/jbc.M210538200

León, G., Holuigue, L., and Jordana, X. (2007). Mitochondrial complex II is essential for gametophyte development in Arabidopsis. Plant Physiol. 143, 1534-1546. doi: 10.1104/pp.106.095158

Logan, D. C., Millar, A. H., Sweetlove, L. J., Hill, S. A., and Leaver, C. J. (2001). Mitochondrial biogenesis during germination in maize embryos. Plant Physiol. 125, 662-672. doi: 10.1104/pp.125.2.662

Marzabal, P., Gas, E., Fontanet, P., Vicente-Carbajosa, J., Torrent, M., and Ludevid, M. D. (2008). The maize Dof protein PBF activates transcription of gammazein during maize seed development. Plant Mol. Biol. 67, 441-454. doi: 10.1007/ s11103-008-9325-5

Millar, A. H., Eubel, H., Jansch, L., Kruft, V., Heazlewood, J. L., and Braun, H. P. (2004). Mitochondrial cytochrome c oxidase and succinate dehydrogenase complexes contain plant specific subunits. Plant Mol. Biol. 56, 77-90. doi: 10.1007/s11103-004-2316-2

Molina, C., and Grotewold, E. (2005). Genome wide analysis of Arabidospis core promoters. BMC Genomics 6:25. doi: 10.1186/1471-2164-6-25

Morton, T., Petricka, J., Corcoran, D. L., Li, S., Winter, C. M., Carda, A., et al. (2014). Paired-end analysis of transcription start sites in Arabidopsis reveals plant-specific promoter signatures. Plant Cell 26, 2746-2760. doi: 10.1105/tpc. 114.125617

Nambara, E., and Marion-Poll, A. (2003). ABA action and interactions in seeds. Trends Plant Sci. 8, 213-217. doi: 10.1016/S1360-1385(03)00060-8

Narsai, R., Law, S. R., Carrie, C., Xu, L., and Whelan, J. (2011). In-depth temporal transcriptome profiling reveals a crucial developmental switch with roles for RNA processing and organelle metabolism that are essential for germination in Arabidopsis. Plant Physiol. 157, 1342-1362. doi: 10.1104/pp.111.183129

Oñate-Sanchez, L., and Vicente-Carbajosa, J. (2008). DNA-free RNA isolation protocols for Arabidopsis thaliana, including seeds and siliques. BMC Res. Notes 1:93. doi: 10.1186/1756-0500-1-93
Parcy, F., Valon, C., Raynal, M., Gaubier-Comella, P., Delseny, M., and Giraudat, J. (1994). Regulation of gene expression programs during Arabidopsis seed development: roles of the ABI3 locus and of endogenous abscisic acid. Plant Cell 6, 1567-1582. doi: 10.1105/tpc.6.11.1567

Roschzttardtz, H., Fuentes, I., Vásquez, M., Corvalán, C., León, G., Gómez, I., et al. (2009). A nuclear gene encoding the iron-sulfur subunit of mitochondrial complex II is regulated by B3 domain transcription factors during seed development in Arabidopsis. Plant Physiol. 150, 84-95. doi: 10.1104/pp.109. 136531

Roussell, D. L., Thompson, D. L., Pallardy, S. G., Miles, D., and Newton, K. J. (1991). Chloroplast structure and function is altered in the NCS2 maize mitochondrial mutant. Plant Physiol. 96, 232-238. doi: 10.1104/pp.96. 1.232

Sandoval, P., León, G., Gómez, I., Carmona, R., Figueroa, P., Holuigue, L., et al. (2004). Transfer of RPS14 and RPL5 from the mitochondrion to the nucleus in grasses. Gene 324, 139-147. doi: 10.1016/j.gene.2003.09.027

Santos-Mendoza, M., Dubreucq, B., Baud, S., Parcy, F., Caboche, M., and Lepiniec, L. (2008). Deciphering gene regulatory networks that control seed development and maturation in Arabidopsis. Plant J. 54, 608-620. doi: 10.1111/ j.1365-313X.2008.03461.x

Schwartz, S. H., Leon-Kloosterziel, K. M., Koornneef, M., and Zeevaart, J. A. (1997). Biochemical characterization of the aba2 and aba3 mutants in Arabidopsis thaliana. Plant Physiol. 114, 161-166. doi: 10.1104/pp.114.1.161

Sew, Y. S., Ströher, E., Holzmann, C., Huang, S., Taylor, N. L., Jordana, X., et al. (2013). Multiplex micro-respiratory measurements of Arabidopsis tissues. New Phytol. 200, 922-932. doi: 10.1111/nph.12394

Sprenger-Haussels, M., and Weisshaar, B. (2000). Transactivation properties of parsley proline-rich bZIP transcription factors. Plant J. 22, 1-8. doi: 10.1046/ j.1365-313x.2000.00687.x

Sun, F., Huo, X., Zhai, Y., Wang, A., Xu, J., Su, D., et al. (2005). Crystal structure of mitocondrial respiratory membrane complex II. Cell 121, 1043-1057. doi: 10.1016/j.cell.2005.05.025

Weitbrecht, K., Müller, K., and Leubner-Metzger, G. (2011). First off the mark: early seed germination. J. Exp. Bot. 62, 3289-3309. doi: 10.1093/jxb/err030

Weltmeier, F., Ehlert, A., Mayer, C. S., Dietrich, K., Wang, X., Schutze, K., et al. (2006). Combinatorial control of Arabidopsis proline dehydrogenase transcription by specific heterodimerisation of bZIP transcription factors. EMBO J. 25, 3133-3143. doi: 10.1038/sj.emboj.7601206

Yankovskaya, V., Horsefield, R., Tornroth, S., Luna-Chavez, C., Miyoshi, H., Leger, C., et al. (2003). Architecture of succinate dehydrogenase and reactive oxygen species generation. Science 299, 700-704. doi: 10.1126/science. 1079605

Yoo, S. D., Cho, Y. H., and Sheen, J. (2007). Arabidopsis mesophyll protoplasts: a versatile cell system for transient gene expression analysis. Nat. Protoc. 2, 1565-1572. doi: 10.1038/nprot.2007.199

Conflict of Interest Statement: The authors declare that the research was conducted in the absence of any commercial or financial relationships that could be construed as a potential conflict of interest.

Copyright (C) 2017 Restovic, Espinoza-Corral, Gómez, Vicente-Carbajosa and Jordana. This is an open-access article distributed under the terms of the Creative Commons Attribution License (CC BY). The use, distribution or reproduction in other forums is permitted, provided the original author(s) or licensor are credited and that the original publication in this journal is cited, in accordance with accepted academic practice. No use, distribution or reproduction is permitted which does not comply with these terms. 